\title{
Metrics for Laparoscopic Skills Trainers: The Weakest Link!
}

\author{
Stephane Cotin ${ }^{1,3}$, Nicholas Stylopoulos ${ }^{1,2,3}$, Mark Ottensmeyer ${ }^{1,3}$, \\ Paul Neumann ${ }^{1,3}$, David Rattner ${ }^{2,3}$, and Steven Dawson ${ }^{1,3}$ \\ 1 The Simulation Group, Massachusetts General Hospital, Boston, MA \\ 2 Department of Surgery, Massachusetts General Hospital, Boston, MA \\ 3 Harvard Medical School, Boston, MA \\ scotin@partners.org
}

\begin{abstract}
Metrics are widely employed in virtual environments and provide a yardstick for performance measurement. The current method of defining metrics for medical simulation remains more an art than a science. Herein, we report a practical scientific approach to defining metrics, specifically aimed at computer-assisted laparoscopic skills training. We also propose a standardized global scoring system usable across different laparoscopic trainers and tasks. The metrics were defined in an explicit way based on the relevant skills that a laparoscopic surgeon should master. We used a five degree of freedom device and a software platform capable of 1) tracking the motion of two laparoscopic instruments 2) real time information processing and feedback provision. A validation study was performed. The results show that our metrics and scoring system represent a technically sound approach that can be easily incorporated in a computerized trainer for any task, enabling a standardized performance assessment method.
\end{abstract}

\section{Introduction}

Ask a surgeon whether virtual reality based simulation will be an important educational aid and the answer is predictably "yes". The rationale for this response is that current methods of training have been recently placed under scrutiny by experts, physicians, and the general public. As a result of the Institute of Medicine report "To Err is Human: Building a Safer Health System" 8, the American Board of Medical Specialties (ABMS) along with the Accreditation Council on Graduate Medical Education (ACGME) initiated a joint outcomes project. The aim of this project is to identify and quantify the factors that constitute "medical competence" and promote the development of the appropriate training models to improve medical performance and skills acquisition [1]. These leading medical educators advocate the application of computer simulation in the arena of medical training. Indeed, during the past ten years medical simulation has progressed to a level of academic prototypes [12,3/18, 4 ] or commercial products [13:14]. Computerized systems have enabled the recording of quantitative parameters including instrument motion and applied forces, which cannot

T. Dohi and R. Kikinis (Eds.): MICCAI 2002, LNCS 2488, pp. 3543 2002.

(C) Springer-Verlag Berlin Heidelberg 2002 
be measured with conventional instruments alone. This is a major step towards skill acquisition. As Dr David Leach, the director of ACGME said "What we measure we tend to improve" [11]. A corollary of this statement is that by creating accurate and relevant metrics we can augment the advantages offered by existing training systems and improve skills acquisition and learning. Unfortunately today, the lack of appropriate metrics represents one of the weakest links in medical simulation.

\subsection{Laparoscopic Skills Training}

The expanding application of laparoscopy is a factor that has reemphasized the absolute requirement for technical competency. Laparoscopic surgery is a technically demanding discipline, which imposes significant psychomotor challenges on surgeons, making it an ideal candidate for medical simulation. However, because of the need for faster and more accurate modelling algorithms, the educational and clinical aspects have been downplayed and several questions remain to be answered. Is task training important? If yes, what tasks should be simulated and what are the appropriate ways to evaluate task performance? These two questions are very familiar to all the researchers in the field of medical simulation, but few have addressed these issues. The first question has been answered by the Society of American Gastrointestinal Surgeons (SAGES), which is the first official association to officially adopt a set of skill testing modules [16]. Skills training is essential because it optimizes the learning opportunities in the operating room by increasing the level of confidence and comfort with the fundamentals of laparoscopy $21,2,5,11$. The second question has been partially answered through the development of virtual environments and enhanced training boxes that permit controlled conditions under which we can elucidate the elements of skill and try to understand the underlying cognitive demands of laparoscopic surgery [20].

Until recently there was a tendency to view performance assessment and metrics in very simplistic terms [11]. The first non-computer based laparoscopic skills trainers incorporated empirical outcome metrics as an indirect way to evaluate performance and learning. Performance, however, cannot be evaluated solely on the basis of the outcome [15]. With the advent of computer simulation it became apparent that it will be possible to define specific metrics that can be used effectively and efficiently to evaluate performance [17]. An efficient metric should not only provide information about performance but also identify 1) the key success/failure factors of performance, 2) the size and the nature of the gap between expert and novice performance. Thus, it should point to the action that needs to be taken in order to resolve these gaps.

\section{Material and Methods}

In order to make computer simulation a stronger link in medical training, we propose a set of appropriate metrics that illustrates the relationship between the kinematic properties of the motion of laparoscopic instruments and the special cognitive and psychomotor skills that a laparoscopic surgeon should master. 
Additionally, a standardized method of performance assessment is imperative to ensure comparability across different systems and training conditions. In this section we first introduce the concept of task-independent metrics for skills assessment and how it relies on kinematics analysis. We describe the various parameters used to represent the essential characteristics of any laparoscopic task, and how these parameters are combined into a standardized score. The mechanical apparatus used to record data from the laparoscopic instruments is detailed in section (2.2), followed by a description of the implementation of the kinematic analysis software and its interface in section (2.3). Finally, the experimental setup and the validation study are described in section (3) as well as the results of the study and their clinical significance.

\subsection{Task-Independent Metrics}

It is not clear how surgeons learn and adapt to the unusual perceptual motor relationships in minimally invasive surgery. A major part of the learning process, however, relies on an apprenticeship model according to which an expert surgeon qualitatively assesses the performance of the novice 1 [15. In order to define a quantitative performance metric that is useful across a large variety of tasks, we looked at the way expert surgeons instruct and comment upon the performance of novices in the operating room. Expert surgeons are able to evaluate the performance of a novice by watching on the operating room monitor the motion of the visible part of the instruments that have been introduced into the abdominal cavity. Based on this information and the outcome of the surgical task, the expert surgeon can characterize qualitatively the overall performance of the novice on each of the key parameters that are required for efficient laparoscopic manipulations.

We identified the following components of a task that account for competence while relying only on instrument motion: compact spatial distribution of the tip of the instrument, smooth motion, good depth perception, response orientation, and ambidexterity. Time to perform the task as well as outcome of the task are two other important aspects of the "success" of a task that we decided to include [15 11 16. Finally, in order to transform these parameters into quantitative metrics, we relied on kinematics analysis theory that has been successfully used in previous work to study psychomotor skills [10]. Most laparoscopic tracking devices or haptic interfaces can provide information about kinematic parameters, in particular: position of a three-dimensional point representing the tip of the instrument, rotation of the instrument about its axis, and degree of opening of the handle. All these variables are time-dependent and we will use the following notations in the remaining of the paper: $[x(t), y(t), z(t)]^{T}$ is the three-dimensional position of the tip of the instrument, and $\theta(t)$ is the rotation of the instrument about its axis. The five kinematic parameters we have defined so far are:

- Time: this is the total time required to perform the task (whether the task was successful or not). It is measured in seconds and represented as $P_{1}=T$.

\footnotetext{
1 The term novice connotes a surgeon in training.
} 
- Path Length: it is the length of the curve described by the tip of the instrument over time. In several tasks, this parameter describes the spatial distribution of the tip of the laparoscopic instrument in the workspace of the task. A compact "distribution" is characteristic of an expert. It is measured in centimeters and represented as $P_{2}$.

$$
P_{2}=\int_{0}^{T} \sqrt{\left(\frac{d x}{d t}\right)^{2}+\left(\frac{d y}{d t}\right)^{2}+\left(\frac{d z}{d t}\right)^{2}} d t
$$

- Motion Smoothness: this parameter is based on the measure of the instantaneous jerk defined as $j=\frac{d^{3} x}{d t^{3}}$ and represents a change of acceleration and is measured in $\mathrm{cm} / \mathrm{s}^{3}$. We derive a measure of the integrated squared jerk $J$ from $j$ as follows:

$$
J=\sqrt{\frac{1}{2} \int_{0}^{T} j^{2} d t}
$$

The time-integrated squared jerk is minimal in smooth movements [6]. Because jerk varies with the duration of the task, $J$ has to be normalized for different tasks durations. This was done by dividing $J$ by the duration $T$ of the task: $P_{3}=J / T$.

- Depth Perception: we measure depth perception as the total distance travelled by the instrument along its axis. It is represented as $P_{4}$ and can easily be derived from $P_{2}$.

- Response orientation: this parameter characterizes the amount of rotation about the axis of the instrument and illustrates the ability to place the tool in the proper orientation in tasks involving grasping, clipping or cutting. It is represented as $P_{5}$ and measured in radians.

$$
P_{5}=\sqrt{\int_{0}^{T} \frac{d \theta^{2}}{d t} d t}
$$

All of these parameters can be seen as cost functions where a lower value describes a better performance. Task-independence is achieved by computing the z-score [7] of each parameter $P_{i}$. The z-score $z_{i}$ corresponding to parameter $P_{i}$ is defined as follows

$$
z_{i}=\frac{P_{i}^{N}-\overline{P_{i}^{E}}}{\sigma_{i}^{E}}
$$

where $\overline{P_{i}^{E}}$ is the mean of $\left\{P_{i}\right\}$ for the expert group and $\sigma_{i}^{E}$ is the standard deviation. $P_{i}^{N}$ corresponds to the result obtained by the novice for the same parameter. Assuming a normal distribution, 95\% of the expert group should have a z-score $z_{i} \in[-2 ; 2]$. Therefore we can limit the range of values for $z_{i}$ to $\left[-z_{\max } ; z_{\max }\right]$ with $z_{\max }>2$. In our implementation we used $z_{\max }=10$.

A standardized score is computed from the independent z-scores $z_{i}$ according to the following equation

$$
z=1-\frac{\sum_{i=1}^{N} \alpha_{i} z_{i}}{\sum_{i=1}^{N} \alpha_{i} z_{\max }}-\alpha_{0} z_{0}
$$


where $N$ is the number of parameters, $z_{0}$ is a measure of the outcome of the task and $\alpha_{0}$ the weight associated with $z_{0}$. There are various ways of evaluating $z_{0}$ and it can be either a binary measure $\left(z_{0}=0\right.$ for success, $z_{0}=1$ for failure), or a more complex measure, as introduced by several authors [15 16]. Similarly, $\alpha_{i}$ is the coefficient for a particular parameter $P_{i}$.

\subsection{Apparatus}

The subjects' position trajectories was measured with a modified Virtual Laparoscopic Interface (VLI) (Immersion Corp., San Jose, CA). The VLI includes tool handles similar to laparoscopic instruments, but there are no tool tips with which to manipulate real objects. To permit the use of the VLI as a tracking instrument, the main shafts and tool handles from the VLI were removed, and replaced with a system which allows the use of a variety of laparoscopic instruments. Figure 1
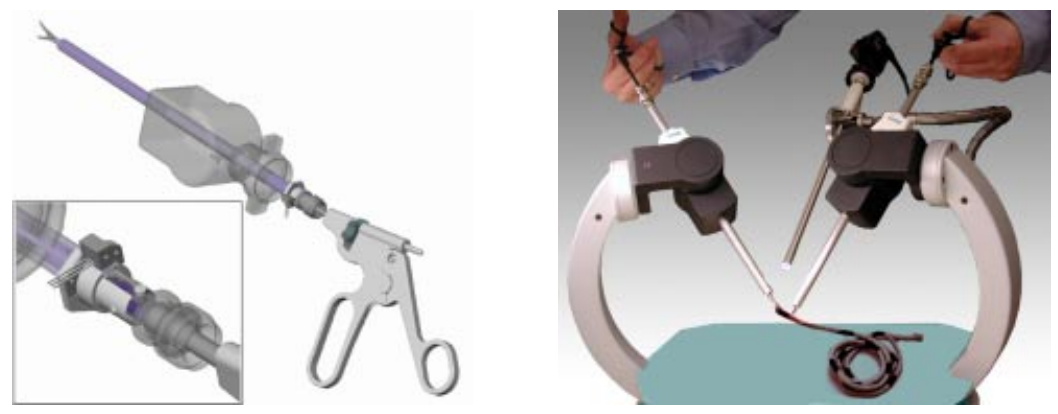

Fig. 1. (Left): modified Virtual Laparoscopic Interface to support the use of laparoscopic instruments and real objects. Inset (left): detail of bayonet connection and Hall effect/magnet arrangement. (Right): testbed for the "Cobra Rope Drill" task.

shows the modified system, including the original VLI equipment, the new main shafts, the laparoscopic instruments, and the bayonet connectors which permit rigid connection between the two. By replacing the main shaft of the VLI and modifying the laparoscopic instruments, the pitch/yaw/roll and thrust sensors can be used without modification. The gripper sensor in the original system is a Hall effect sensor that detects the motion of the actuator shaft in the tool handle. The original sensor blocks the insertion of full length instruments, so it was removed, and a new Hall sensor and rare-earth magnet combination were installed as shown in figure 1 This also simplifies interfacing the modifications to the VLI, since the signal output is electrically identical to the unmodified system.

The instrument modifications and Hall effect sensor placement were performed as precisely as possible, but due to the sensitivity of the sensor to small changes in the distance between it and the magnet, each instrument was separately calibrated. This ensures good correspondence between the true gripper axis motion and that measured by the system. Changing the calibration constants and offsets when switching tools is computed by software. 
Laparoscopic trocar and cannula assemblies were also incorporated and special care was taken so that the friction at the point of entry of the instruments is realistic. For visual feedback we used a fixed surgical endoscope, camera, and light source (Telecam SL NTSC/Xenon 175, Karl Storz Endoscopy-America, Inc., Culver City, CA), to provide the same visual feedback encountered in minimally invasive procedures. The relative orientation between the endoscopic camera, the instrument axis and the monitor was approximately the same to minimize the mental effort of relating visual and instrument frames [19].

\subsection{Software System}

We have developed a software interface that integrates data processing as well as visualization of the instrument motion, path, parameters $P_{i}$ and normalized score. Our system uses the Virtual Laparoscopic Interface API from Immersion Corp. as a basis for communication with the VLI. The raw data consists of timestamped values of the position and orientation of each of the two laparoscopic instruments, recorded at a sampling rate of about $20 \mathrm{~ms}$. Before computing any of the kinematic parameters, the raw data is filtered. We have implemented various low-pass filters, using mean and median filtering. The results presented in section (3) have been obtained after filtering with a median filter (window size $=5)$. In order to compute accurately high order derivatives of the position, we used a second-order central difference method (if no filtering and/or first order methods were used, an accurate computation of the jerk would be very difficult).

We implemented the user interface using $\mathrm{C}++$, FLTK2 2 , and OpenGL. The user interface offers real-time display of the tip of the tool, and its path (see Figure 22). Kinematics analysis and computation of the score are performed at the end of the task, providing immediate information to the user. Moreover, a visual comparison of the results of the experts group and the novice illustrates clearly what skills need to be improved in order to get a higher score. To account for the two laparoscopic instruments available in the VLI, we compute a z-score for each tool: $z_{\text {left }}$ and $z_{\text {right }}$, and define the overall score as $z=\left(z_{\text {left }}+z_{\text {right }}\right) / 2$. The coefficients $\alpha_{i}$ are all set to 1 except for $z_{0}$ which is set to 0.5 if the goal of the task is not achieved. This illustrates the flexibility of our approach and its ability to take into account the specifics of the hardware and goals of the tasks (by allowing one to vary the weights $\alpha_{i}$ of the parameters $P_{i}$ ).

\section{Experimental Results and Discussion}

Three tasks of increasing difficulty selected from established training programs (the Yale Laparoscopic Skills and Suturing Program, the SAGES-FLS training program and the graded exercises used at Harvard Center of Minimally Invasive Surgery) were examined:

\footnotetext{
${ }^{2}$ Fast Light Toolkit, http://www.fltk.org
} 
- Task 1: Peg Board Transfer: the purpose of this task is to assess eye-hand coordination, bimanual dexterity, and depth perception,

- Task 2: Cobra Rope Drill: the purpose of this task is to assess depth perception, two-handed choreography, and non-dominant hand development,

- Task 3: Needle-Cap : the purpose of this task is to assess depth perception, non-dominant hand development, and targeting.

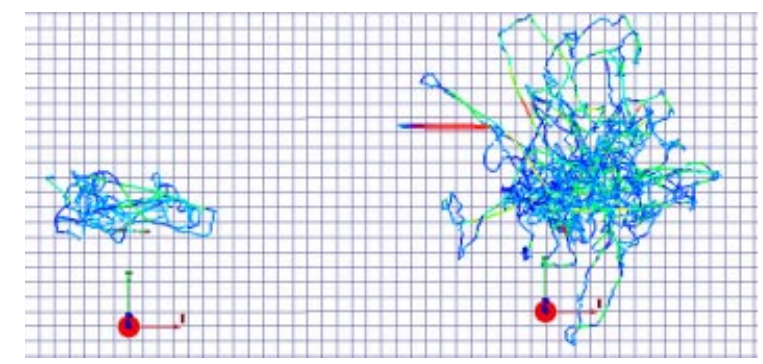

Fig. 2. Screenshot of our user interface displaying the path of the expert (left) and the novice (right) after completion of the "Cobra Rope Drill" task. The trajectory is color-coded based on velocity. A compact path is characteristic of expert's performance.

In order to validate our scoring system, we conducted a study comparing experts and novices surgeons. The expert group consisted of staff surgeons from our hospital, while the novice group consisted of 20 surgeons in training. Each of the experts was asked to perform each task several times. Each of the novices was asked to perform each task once, and was visually assessed by an expert. Most of the novices had had prior exposure to the tasks they were asked to perform. The values of the different parameters $\left\{P_{i}\right\}$ as well as the score $z$ were computed using our software platform and recorded on file.

The results of the study illustrate several important aspects of our method. First, they confirm that our metrics are independent of the task being performed. Without changing any of the parameters $P_{i}$ or weights $\alpha_{i}$ used in the computation of the overall score, our method still provides an efficient way of discriminating between expert and novice performance, irrespective of the task, as illustrated in table (11).

Table 1. This table presents the overall scores obtained by novices and experts in our study. It clearly highlights the gap existing between expert's and trainee's performance, irrespective of the task. None of the subjects in our novice group obtained a score above the threshold $\left(\overline{z^{E}}-2 \sigma^{E}\right)$ that distinguishes experts from non-experts.

\begin{tabular}{l|c|c|c} 
& Task 1 & Task 2 & Task 3 \\
\hline Expert mean score (standard deviation) & $1.0(0.035)$ & $1.0(0.03)$ & $1.0(0.015)$ \\
\hline Novice minimum score & 0.22 & 0.38 & 0.04 \\
\hline Novice maximum score & 0.69 & 0.58 & 0.72
\end{tabular}

In addition to an overall measure that discriminates between experts and nonexperts, our method provides additional feedback by identifying the key factors 
that contribute to the overall score. For instance, we can see in Figure (3) that factors such as depth perception, smoothness of motion, and response orientation are major indicators of performance, while time and path length (often referenced in the literature) do not provide enough information to capture the magnitude of the difference. By comparing the expert's verbal assessment and the parameters $P_{i}$ for which existed a significant difference between novice and expert, a high correlation was found, thus validating our choice of parameters.

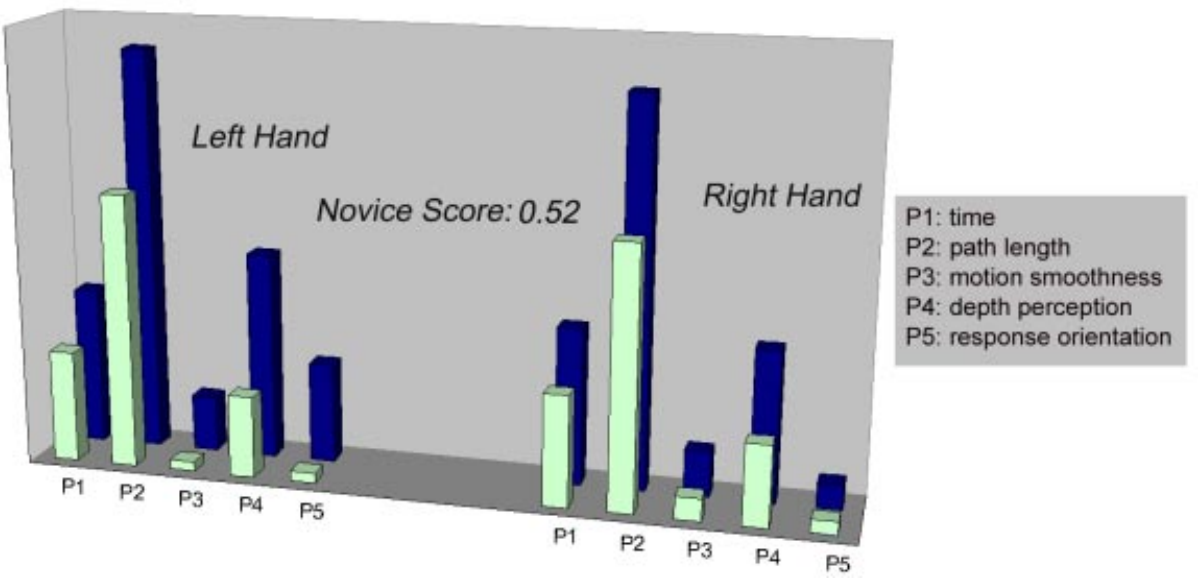

Fig. 3. This diagram illustrates the comparison between the experts group (light blue) and a novice (dark blue). The overall score given to the novice by our system was 0.52 ; this score can be explained by low values associated with particular parameters $\left(P_{3}, P_{4}, P_{5}\right)$. Such feedback is very important to provide a meaningful interpretation of the score.

\section{Conclusion}

The method presented in this paper represents a work in progress. We have demonstrated that it is possible to extract meaningful information from the analysis of the motion of laparoscopic instruments only. This is a novel contribution and it allows the definition of task-independent metrics and the formulation of a standardized global scoring system for laparoscopic skills training. We have also shown that our approach can be a successful alternative to the verbal assessment scheme used in the classic apprenticeship model. Therefore it can be introduced in computer-based learning systems for surgical skills training.

\section{Acknowledgements}

This work was supported by U.S. Army Medical Research Acquisition Activity under contract DAMD17-99-2-9001. The ideas and opinions presented in this paper represent the views of the authors and do not, necessarily, represent the views of the Department of Defense. 


\section{References}

1. ACGME Outcome project. Available at http://www.acgme.org/outcome/

2. Anastakis, D.J., Hamstra, S.J., Matsumoto, E.D.: Visual-spatial abilities in surgical training. Am. J. Surg. 179(6) (2000) 469-471

3. Brown, J., Montgomery, K., Latombe, J.C., Stephanides, M.: A Microsurgery Simulation System. In: Niessen, W.J., Vierger, M.A. (eds.): MICCAI 2001, vol. 2208. Springer-Verlag Berlin Heidelberg (2001) 137-144

4. Cotin, S., Delingette, H., Ayache, N.: A hybrid elastic model for real-time cutting, deformations and force feedback for surgery training and simulation. The Visual Computer 16 (2000) 437-452

5. Derossis, A.M., Fried, G.M., Abrahamowicz, M., Sigman, H.H., Barkun, J.S., Meakins, J.L.: Development of a model for training and evaluation of laparoscopic skills. Am. J.Surg. 175(6) (1998) 482-487

6. Hogan, N., and Flash, T.: Moving gracefully: Quantitative theories of motor coordination. Trends Neurosci. 10 (1987) 170-174

7. Howell D.C. (ed.). Fundamental Statistics for the Behavioral Sciences with CDRom. Duxbury Press Pacific Grove, CA (1998)

8. Kohn, L.T., Corrigan, J.M., Donaldson, M.F. (eds.): To Err is Human. Building a Safer Health System. Institute of Medicine, National Academy Press, Washington, D.C. (1999)

9. Kuhnapfel, U.G., Kuhn, C., Hubner, M., Krumm, H.G., Maa, E.H., Neisius, B.: The Karlsruhe Endoscopic Surgery Trainer as an example for virtual reality in medical education, Minimally Invasive Therapy and Allied Technologies 6 (1997) 122-125

10. Mavrogiorgou, P., Mergl, R., et al.: Kinematic analysis of handwriting movements in patients with obsessive-compulsive disorder. J. Neurol. Neurosurg. Psychiatry 70(5) (2001) 605-612

11. Metrics for objective assessment of surgical skills workshop. Scottsdale Arizona (2001). Final report. Available at: http://www.tatrc.org/

12. Picinbono, G., Delingette, H., Ayache, N. Non-linear and anisotropic elastic soft tissue models for medical simulation. In ICRA2001: IEEE International Conference Robotics and Automation, (2001)

13. Procedicus MIST. Mentice Medical Simulation, Gothenburg, Sweden

14. Reachin Laparoscopic Trainer. Reachin Technologies AB. Stockholm, Sweden

15. Rosser, J.C. Jr., Rosser, L.E., Savalgi, R.S.: Objective evaluation of a laparoscopic surgical skill program for residents and senior surgeons. Arch. Surg. 133(6) (1998) 657-661

16. Society of American Gastrointestinal Endoscopic Surgeons. Fundamentals of laparoscopic surgery. Available at: http://www.fls-test.org/

17. Satava, R.M.: Accomplishments and challenges of surgical simulation. Surg. Endosc. 15(3) (2001) 232-241

18. Szekely, G., Brechbuhler, C., Hutter, R., Rhomberg, A., Ironmonger, N. Schmid, P.: Modelling of Soft Tissue Deformation for Laparoscopic Surgery Simulation. In: Wells, W.M., Colchester, A., Delp S. (eds.): MICCAI 1998. Lecture Notes in Computer Science, vol. 1496. Springer-Verlag Berlin Heidelberg (1998) 550-561

19. Tendick, F., Jennings, R., Tharp, G., Stark, L.: Sensing and Manipulation Problems in Endoscopic Surgery: Experiment, Analysis, and Observation. Presence 2(1) (1993) 66-81

20. Tendick, F., Downes, M., Goktekin, T., Cavusoglu, M.C., Feygin, D., Wu, X., Eyal, R., Hegarty, M., Way L.W.: A Virtual Environment Testbed for Training Laparoscopic Surgical Skills. Presence 9(3) (2000) 236-255

21. Wanzel, K.R., Hamstra, S.J., Anastakis, D.J., Matsumoto, E.D., Cusimano, M.D.: Effect of visual-spatial ability on learning of spatially-complex surgical skills. Lancet 359(9302) (2002) 230-231 